\title{
Recovery of left ventricular function and sleep apnoea after acute myocardial infarction
}

\author{
From the authors:
}

We are pleased to have the opportunity to compare our findings [1] with the similar observational study in patients with acute coronary syndrome by SCHIzA et al. [2]. Since the two studies were published almost simultaneously in 2012 there has not yet been the opportunity to do so.

In both studies the natural course of polysomnographically assessed sleep apnoea was observed within the first months after acute coronary syndrome [2] and acute myocardial infarction [1]. At baseline, average left ventricular function was mildly impaired in both study populations (left ventricular ejection fraction (LVEF) $47 \pm 10 \%$ [2] and $44 \pm 2 \%$ [1]). Importantly, in the study by ScHIzA et al. [2] patients with large myocardial infarctions were systematically excluded (LVEF $<40 \%$ ), resulting in a patient population without major impairment of left ventricular function and a smaller range of severity of heart failure. Both studies found a very high prevalence of sleep apnoea within the first days after the acute coronary event (apnoea/hypopnoea index (AHI) $\geqslant 10$ per hour: 54\% [2] and AHI $\geqslant 15$ per hour: 55\% [1]) and a decrease of AHI over time after the coronary event, which was mainly due to a reduction in obstructive apnoeas and hypopnoeas [1, 2].

While our last follow-up polysomnography was at 3 months, ScHIZA et al. [2] extended such findings with a second follow-up polysomnography at 6 months, demonstrating that there is also a progressive decrease of $\mathrm{AHI}$ and an increase of sleep efficiency from 1 to 6 months after the acute coronary syndrome. In our study [1] cardiovascular magnetic resonance imaging (MRI), as the accepted gold standard for the assessment of left ventricular systolic function, was performed to 1) determine LVEF with the lowest possible variability of measurement [3], and 2) to relate changes in the severity of AHI to changes in LVEF. The striking finding was that the improvement of AHI was strongly linked to an improvement of cardiac function [1], suggesting an important mechanism of how sleep disordered breathing may improve after an acute myocardial infarction. The finding was consistent when comparing the groups of patients improving LVEF by $\geqslant 5 \%$ within 3 months versus patients with less improved LVEF $(<5 \%)$ or when analysed in a regression analysis with LVEF and AHI as continuous variables ( $\beta$ coefficient $0.37,95 \%$ CI $0.17-1.69 ; p=0.018$ ). We admit that the cut-off (change of LVEF $\geqslant 5 \%$ versus $<5 \%$ ) for the stratified analysis was somewhat arbitrary, however, the stratified analysis helps to communicate the important clinical implication to the reader: "Of those patients who improved their cardiac function $(\triangle \mathrm{LVEF} \geqslant 5 \%)$ within the first 12 weeks after acute myocardial function, a substantial proportion $(31 \%)$ shifted from moderate-to-severe sleep apnoea (AHI $\geqslant 15$ per hour) to no or mild sleep apnoea (AHI $<15$ per hour) with no or uncertain indication for treatment" [1]. Conversely, worsening of cardiac function should certainly also lead to reevaluation of sleep apnoea, since therapy may be indicated or positive airway pressure (PAP) treatment may need to be adjusted.

MERmigkis et al. [4] state that they did not find such a relationship between improvement of LVEF and AHI in their study population. In our opinion this finding does not contradict our findings due to two reasons: 1) SCHIZA et al. [2] included only patients with "preserved" left ventricular function (LVEF $\geqslant 40 \%$ ). Limiting the range of LVEF to patients with only mildly impaired or normal LVEF the potential for improvement of LVEF is limited. Therefore, our study samples were not comparable in this respect. 2) If you intend to demonstrate an effect on LVEF, studies using echocardiography require a seven-fold larger sample size compared to studies using cardiovascular MRI [3]. The major reason for this is that the variability of measurement is significantly lower using cardiovascular MRI [3], which is reflected by the given standard deviations of baseline LVEF in the studies of SCHIZA et al. [2] and our study [1] (47 $\pm 10 \%$ and $44 \pm 2 \%$, respectively). Therefore, the statistical significance of change in LVEF cannot be compared between studies of similar size when using different techniques to assess LVEF.

The second comment by MERMigkis et al. [4] refers to the similarly low sleep efficiency reported in our sample of patients $<5$ days and 3 months after acute myocardial infarction with LVEF improvement (76 versus $74 \%$ ) and without LVEF improvement (70 versus 70\%). In contrast, SCHIZA et al. [2] reported a plausible progressive rise and normalisation of sleep efficiency from 3 days to 6 months after the acute coronary syndrome $(62 \%$ to $84 \%)$, which parallels the improvement of sleep apnoea. The explanation for the sustained low sleep efficiency in our patients with acute myocardial infarction and heart failure (mean 
$\mathrm{N}$-terminal pro-brain natriuretic peptide $1572 \mathrm{pg} \cdot \mathrm{mL}^{-1}$ ) is most likely multifactorial, as described in a recent publication by our group [5]. The similarly low sleep efficiency in patients with chronic heart failure could not be explained by arousals from sleep apnoea or periodic limb movements alone [5]. Other factors such as the severity of heart failure and (mood) depression after a severe life threatening event may also play an important role. In patients with chronic heart failure impaired sleep efficiency (assessed by polysomnography) was strongly associated with impaired survival [5]. In this context, we believe that MERMIGKIs et al. [4] have raised a very important point, that further research on sleep efficiency or other measures of sleep quality and cardiac outcome is required.

To attenuate concerns that in our study [1] the sleep apnoea severity in the LVEF improved and LVEF unchanged groups was due to differences in sleep position and sleep stage we can provide the following data, which were in part presented in the manuscript [1]. Both time in the supine position and time in rapid eye movement (REM) sleep at baseline (43 versus 39\% of total sleep time and 17 versus $16 \%$ of total sleep time) as well as changes of time in the supine position or in REM sleep over time were similar between groups ( $\mathrm{p}=0.150$ and $\mathrm{p}=0.757$, respectively).

As discussed in the manuscript [1] the nonsignificant fall of body weight in the LVEF improved group and the rise in body weight of the LVEF unchanged group (between group difference $p=0.062$ ), which most likely reflects changes of fluid overload or content, may in part explain the results of our study. However, the study was not designed to address such pathomechanisms of sleep apnoea. The absence of changes in body weight in the report of SCHIZA et al. [2] may be a consequence of the lower severity of heart failure by exclusion of patients with LVEF $<40 \%$ and the analysis of the entire sample without stratification.

Both studies confirm a strikingly high prevalence of moderate-to-severe sleep apnoea early after acute coronary syndrome or acute myocardial infarction, and a progressive improvement of sleep apnoea in some of the patients early after such an event. To date diagnosis or treatment of sleep apnoea is not part of the recommended or standard care of patients early after acute myocardial infarction [6]. Since sleep apnoea is associated with increased cardiac workload [7] and myocardial damage [8] in the early phase after myocardial infarction, intervention trials are warranted to assess the safety and efficacy of PAP treatment in this time period.

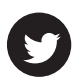

@ERSpublications

Recovery of left ventricular function after acute myocardial infarction leads to attenuation of sleep apnoea http://ow.ly/kslug

Tanja Greimel, Stefan Buchner and Michael Arzt

Klinik und Poliklinik für Innere Medizin II, Universitätsklinikum Regensburg, Regensburg, Germany.

Correspondence: M. Arzt, Center for Sleep Medicine, Dept of Internal Medicine II, University Hospital Regensburg, Franz-Josef-Strauss-Allee 11, 93053 Regensburg, Germany. E-mail: michael.arzt@ukr.de

Received: Jan 022013 | Accepted: Jan 052013

Conflict of interest: Disclosures can be found alongside the online version of this article at www.erj.ersjournals.com

\section{References}

1 Buchner S, Greimel T, Hetzenecker A, et al. Natural course of sleep-disordered breathing after acute myocardial infarction. Eur Respir J 2012; 40: 1173-1179.

2 Schiza SE, Simantirakis E, Bouloukaki I, et al. Sleep disordered breathing in patients with acute coronary syndromes. J Clin Sleep Med 2012; 8: 21-26.

3 Bellenger NG, Davies LC, Francis JM, et al. Reduction in sample size for studies of remodeling in heart failure by the use of cardiovascular magnetic resonance. J Cardiovasc Magn Reson 2000; 2: 271-278.

4 Mermigkis C, Bouloukaki I, Schiza SE. Natural course of sleep disordered breathing after acute myocardial infarction. Eur Respir J 2013; 41: 1238-1239.

5 Reinhard W, Plappert N, Zeman F, et al. Prognostic impact of sleep duration and sleep efficiency on mortality in patients with chronic heart failure. Sleep Med 2013 [in press DOI: 10.1016/j.sleep.2012.12.014].

6 Steg PG, James SK, Atar D, et al. ESC Guidelines for the management of acute myocardial infarction in patients presenting with ST-segment elevation. Eur Heart J 2012; 33: 2569-2619.

7 Hetzenecker A, Buchner S, Greimel T, et al. Cardiac workload in patients with sleep-disordered breathing early after acute myocardial infarction. Chest 2012 [in press DOI:10.1378/chest.12-1930].

8 Nakashima H, Katayama T, Takagi C, et al. Obstructive sleep apnoea inhibits the recovery of left ventricular function in patients with acute myocardial infarction. Eur Heart J 2006; 27: 2317-2322. 